\title{
Characterization of Pain, Disability, and Psychological Burden in Marfan Syndrome
}

\author{
Traci J. Speed ${ }^{1,{ }^{*}}$, Vani A. Mathur ${ }^{2}$, Matthew Hand ${ }^{1}$, Bryt Christensen ${ }^{3}$, Paul D. Sponseller ${ }^{4}$, \\ Kayode A. Williams ${ }^{3}$, and Claudia M. Campbell ${ }^{1}$ \\ ${ }^{1}$ Department of Psychiatry and Behavioral Sciences, Johns Hopkins University School of \\ Medicine, Baltimore, Maryland ${ }^{2}$ Department of Psychology, Texas A\&M University, College \\ Station, Texas ${ }^{3}$ Division Pain Medicine, Department of Anesthesiology and Critical Care Medicine, \\ Johns Hopkins University School of Medicine, Baltimore, Maryland ${ }^{4}$ Department of Orthopaedic \\ Surgery, Johns Hopkins University School of Medicine, Baltimore, Maryland
}

\section{Abstract}

The clinical manifestations of Marfan syndrome frequently cause pain. This study aimed to characterize pain in a cohort of adults with Marfan syndrome and investigate demographic, physical, and psychological factors associated with pain and pain-related disability. Two hundred and forty-five participants (73\% female, $89 \%$ non-Hispanic white, $90 \%$ North American) completed an online questionnaire assessing clinical features of Marfan syndrome, pain severity, pain-related disability, physical and mental health, depressive symptoms, pain catastrophizing, and insomnia. Eighty-nine percent of respondents reported having pain with $28 \%$ of individuals reporting pain as a presenting symptom of Marfan syndrome. Almost half of individuals reported that pain has spread from its initial site. Participants in our study reported poor physical and mental health functioning, moderate pain-related disability, and mild levels of depressive symptoms, sleep disturbances, and pain catastrophizing. Those who identified pain as an initial symptom of Marfan syndrome and those who reported that pain had spread from its initial site reported greater psychological burden compared with those without pain as an initial symptom or pain spreading. Physical health is the largest predictor of pain severity and pain-related disability. While pain catastrophizing and worse mental health functioning are significant correlates of pain severity and pain-related disability, respectively. Pain is a significant and persistent problem in Marfan syndrome and is associated with profound disability and psychological burden. Further studies are indicated to better characterize the directionality of pain, pain-related disability, and psychological burden in Marfan syndrome.

\section{Keywords}

Marfan syndrome; pain; quality of life; catastrophizing; questionnaire

*Correspondence to: Traci J. Speed, Johns Hopkins Bayview Medical Campus, 5510 Nathan Shock Drive Suite 100, Baltimore, MD 21224, speed@jhmi.edu.

Conflicts of interest: The authors report no relevant conflict of interests. 


\section{INTRODUCTION}

Marfan syndrome (MFS) is a connective tissue disorder with autosomal dominant inheritance, a prevalence of 1 in 5,000 individuals, and no predilection for geography, gender, or race [Iams, 2010]. The diagnosis of MFS is based on the revised Ghent criteria [Loeys et al., 2010] and is complex due to high phenotypic variability. FBN1 gene mutations lead to connective tissue abnormalities throughout the body with manifestations in almost all physiological systems [Dietz and Pyeritz, 1995]. Cardiovascular, pulmonary, ocular, and musculoskeletal manifestations of the disorder are common and debilitating [Grahame and Pyeritz, 1995; Ramirez and Dietz, 2007; Pearson et al., 2008; von Kodolitsch et al., 2015]. Such multisystem involvement has profound consequences including reduced quality of life [Rao et al., 2016], modified lifestyle [Peters et al., 2001a], early retirement [Velvin et al., 2015b], and severe fatigue [Bathen et al., 2014]. Cardiovascular manifestations, including aortic dilatation, valvular heart disease, left ventricular dilatation, and heart failure are potentially life threatening, and thus have been the primary focus of research in MFS. With advancements in medical and surgical management leading to an increase in life expectancy of 30 years [Silverman et al., 1995; Willis et al., 2009] research efforts have shifted focus towards the burden of MFS on daily functioning.

Pain symptoms are common in MFS, with prevalence ranging from $47 \%$ to $91.5 \%$ [Peters et al., 2001b; Nelson et al., 2015; Velvin et al., 2016]. Pain symptoms may arise from myriad sources including but not limited to arthritis, chest deformities, scoliosis, dural ectasia, and bony overgrowth [Grahame and Pyeritz, 1995; Foran et al., 2005; Dean, 2007; Hasan et al., 2007]. A recent study from Nelson et al. [2015] reported that the majority of individuals with MFS have daily pain, which is frequently undertreated. Not surprisingly, older patients report greater pain severity and decreased physical functioning [Rand-Hendriksen et al., 2010]. Yet, to our knowledge, no study has inquired about pain symptoms over the course of MFS.

While the medical complications, genetic etiologies, and medical management of MFS have been extensively researched, the pain symptoms and psychological factors of MFS that affect quality of life and disability have only been superficially investigated and most findings are from small studies with low response rates. It has long been recognized that individuals with MFS view the disorder as a burden [Van Tongerloo and De Paepe, 1998] with significant negative consequences on their lives [Peters et al., 2001b]. Decreased quality of life is frequently reported in individuals with MFS [Peters et al., 2001a; De Bie et al., 2004; Foran, et al., 2005; Velvin et al., 2015a]. Interestingly, one small study ( $\mathrm{N}=84$ adults) has shown that reduced quality of life did not correlate with the physical symptoms (i.e., aortic dilatation, dural ectasia, and musculoskeletal criteria) of MFS [Rand-Hendriksen et al., 2010]. Psychological burden in MFS has been associated with the subjective perception of severity of disease rather than the objective physical severity of the disease [De Bie et al., 2004]. With advances in medical management and surgical techniques leading to improved longevity in individuals with MFS, it is essential to investigate the role of pain, quality of life, and psychological factors in patients with MFS. The overall purpose of this study was to characterize pain and pain-related disability in a large cohort of individuals 
with MFS and investigate socio-demographic, physical, and psychological factors associated with pain and pain-related disability.

\section{MATERIALS AND METHODS}

\section{Survey/Data Collection}

Before the final survey was hosted on The Marfan Foundation website (http:// www.marfan.org), appropriate approval was obtained by the Johns Hopkins University Institutional Review Board. The study was advertised as a survey for all patients with MFS, regardless of whether they had pain. The survey was maintained on the website from November 2013 to February 2015.

The web-based survey included six sections of 101 total items. Sections included questions about demographics; diagnostic information; common symptoms and features of pain in MFS, change in pain symptoms over time; current symptoms; and quality of life.

\section{Participants}

Four hundred and forty-one participants completed the survey over a 15-month period. Given survey anonymity, all data were self-report and were not verified by examination or use of medical records. Confirmation of MFS diagnosis was based on self-report questions including if the individual was diagnosed with MFS, how MFS was diagnosed, and if there is a family history. Inclusion criteria were the following: (i) report of personal history of MFS; (ii) report of family history of MFS [Loeys et al., 2010]; and (iii) report that MFS was diagnosed by physical exam, genetic testing, ophthalmologic exam, and/or cardiac exam. Respondents were excluded if they did not indicate having MFS (88 individuals), report a family history of MFS (86 individuals), or report how diagnosis was made (22 individuals). Seventy-five percent of respondents reported a diagnosis by physical exam; $31.4 \%$ reported genetic testing; $25.7 \%$ reported cardiac exam (including imaging); $8.2 \%$ reported ophthalmologic exam. Thus, 245 respondents met criteria and were included in analyses.

\section{Demographics}

Participants were asked to self-report their sex, age, race, nationality, and employment status. The majority of respondents were from North America (90.2\%). Additional respondents reported residence in Europe (4.5\%), Australia (2\%), South America (1.6\%), Africa (0.8\%), and Asia (0.4\%).

\section{Self-Reported Clinical Information}

Thirty-seven symptoms associated with MFS were included in the survey (Table I). Participants were asked about initial and current pain symptoms including locations of pain, if pain has spread, symptoms associated with pain, quality of pain, and pain severity and intensity. They also reported treatments they have received for pain.

Pain severity.-Self-reported clinical pain severity was assessed as the average of patients' ratings of "current pain," "pain at its worst," "least pain," and "general pain." Respondents completed a 5-point scale used to denote increasing pain severities with anchor 
labels of (1) mild to (5) excruciating [Hjermstad et al., 2011]. This type of categorical scale is considered ordinal data [Dijkers, 2010].

Numeric rating scale for pain (NRS).-A unidimensional measure of pain in which participants select a whole number that best reflects the intensity of pain [Jensen and McFarland, 1993; Farrar etal.,2001] within the past 7 days. The pain NRS is a single 11point numeric scale anchored by terms describing pain intensity with $0=$ no pain and $10=$ worst pain imaginable. The NRS was also used to retrospectively report pain when it first began. Spearman correlation between the categorical and current NRS was $\mathrm{r}_{\mathrm{S}}=0.76, P<$ $0.001)$.

Oswestry disability index (ODI).--Version 1 of the ODI was included as a wellvalidated measure of disability. The 10-item ODI assesses pain intensity, sleep quality, social life, sexual function, as well as the ability to lift, care for oneself, walk, sit, stand, and travel on a 6-point Likert scale (verbal descriptions of $0=$ least disability to $5=$ most disability). The total score is calculated as a percentage ranging from 0 to 50 , with higher scores indicating more pain-related disability [Fairbank, 2000].

Short form-12 (SF-12).- The SF-12 is a well-validated 12-item questionnaire that assess physical and mental health status. The scale yields two summary scores-mental health (MCS12) and physical health (PCS12) — which are calculated as age-specific mean difference scores and then linearly transformed with respect to the general population (with $\mathrm{M}=50$ and $\mathrm{SD}=10$ ). Higher scores in each category indicate a healthier state [Ware et al., 1996; Jenkinson et al., 1997].

Beck depression inventory-II (BDI).-The BDI is a well-studied and validated, 21-item questionnaire that assess the intensity of depressive symptoms over the past week using a four point Likert scale ( $0-3$, least to highest severity). The total score can range from 0 to 63. Higher scores indicate more depressive symptoms [Beck et al., 1988].

Pain catastrophizing scale (PCS).-The PCS assesses exaggerated negative cognitive and affective responses to pain using 13 items rated on a 5-point scale (0-not at all to 4all the time) with higher scores indicating greater pain catastrophizing [Sullivan et al., 1995].

Insomnia severity index (ISI).--The ISI is a validated, 7-item questionnaire that uses a five point Likert scale to assess the nature, severity, and impact of insomnia [Yu, 2010]. Higher scores indicate greater sleeping difficulties.

\section{Data Analysis}

Descriptive statistics are used to summarize demographic characteristics and frequency of physical symptoms. The Jeffreys method was used to calculate binomial confidence limits for the population [DasGupta et al., 2001]. NRS ratings by MFS symptom, are reported as mean and standard deviation and median with interquartile range (IQR), and compared between groups using the Mann-Whitney U test. Effect size was provided as the difference between median and the $95 \%$ confidence interval of the difference as determined by the Hodges-Lehman Median Difference. Comparisons of pain severity and physical and 
psychological factors by group are reported as median and IQR, and compared between groups using the Mann-Whitney U test. Spearman bivariate correlations were conducted to examine the association between psychological measures and pain characteristics. Finally, hierarchical linear regression was conducted in order to evaluate psychological predictors of pain severity and pain-related disability in MFS. Demographic variables were entered in block one, physical, and pain components were entered in block two, and psychological variables were entered in the third and final block. Data were analyzed using SPSS (IBM statistics, Version 24). As an adjustment for multiple comparisons, a significance level of $<0.01$ was used.

\section{RESULTS}

\section{Participants}

Participant $(\mathrm{N}=245)$ demographic, medical information, and MFS-specific symptoms are reported in Tables I and II, respectively.

\section{Initial Pain}

Most respondents (89.0\%) reported experiencing pain. Pain most frequently began in the back $(50.6 \%)$ and $35.1 \%$ of patients reported that pain began in multiple locations simultaneously. Respondents recalled experiencing a moderate degree of pain at onset (5.3, $\mathrm{SD}=2.4$ ). Despite the high prevalence of pain symptoms, $41.0 \%$ reported never receiving a pain diagnosis. Respondents described pain at onset most frequently as aching, sharp, stiff, or throbbing.

\section{Pain Spreading}

Forty-four percent of individuals with initial pain reported that pain spread to other locations, with $64 \%$ reporting spread to more than one location (average of 2.5 locations, SD $=1.6$ ), and $16.7 \%$ indicated progression to widespread pain. The most common symptoms associated with both initial and pain spreading were stiffness, difficulty walking, muscle spasms, and muscle weakness.

\section{Current Pain}

At the time of survey completion, respondents reported an average pain rating of 4.4 (SD = 2.7). Although still a moderate level of pain, current pain was rated less intense compared to initial pain levels $(P<0.001)$. Respondents diagnosed with kyphosis, degenerative disc disease, early osteoarthritis, and dural ectasia reported greater current pain levels compared with those without these diagnoses $(P \mathrm{~S} \leq 0.01)$. Those with joint hypermobility and scoliosis did not report greater current pain levels compared to those without respective symptoms as reported in Table III.

Of respondents experiencing pain, two thirds (66.5\%) reported that over the 7 days prior to survey completion, pain occurred greater than $50 \%$ percent of the time with the majority of participants describing pain as discomforting or burdening. As $90.3 \%$ reported experiencing sudden increases in pain with $76.4 \%$ describing their worst pain as horrible or excruciating. Physical stress was the most common trigger of pain exacerbation followed by standing, 
work, exercise, cold weather, emotional stress, and sitting. The majority of respondents, $70.3 \%$, reported that pain has never remitted since its onset, and only $46.6 \%$ of respondents were satisfied with their current pain management. The symptoms associated with current pain are consistent with reports of initial pain including stiffness, difficulty walking, muscle spasms, weakness and increased sensitivity; thus these symptoms do not appear to change over time. Pain quality was also described similarly between onset and current pain; specifically described as aching, sharp, stiff, dull, or throbbing.

\section{Psychological Burden}

Assessment of pain severity, pain-related disability, physical functioning, and psychological factors including mental health functioning, pain catastrophizing, insomnia, and depressive symptoms are reported in Table IV, reported by pain subgroup. Physical and mental health scores were lower compared to the US national average of 50 with a standard deviation of 10 for both scales [Gandek et al., 1998] and consistent with previous MFS studies [RandHendriksen et al., 2010]. Participants in our study reported mild levels of depressive symptoms, sleep disturbances, and pain catastrophizing. Participants exhibited a moderate level of pain-related disability. Those who identified pain as an initial symptom of MFS reported significantly worse pain-related disability, pain catastrophizing, and depressive symptoms compared to individuals who did not have pain at onset of MFS. Compared with those without spreading, those individuals reporting that pain had spread from its initial location reported significantly worse pain severity, pain-related disability, physical health functioning, pain catastrophizing, sleep disturbances, and depressive symptoms $\left(P_{\mathrm{S}} \leq 0.01\right)$. Individuals with pain reported poor mental health functioning, without differences between the various pain sub-groups. The unemployed had significantly greater severity across all psychological factors.

Table V shows correlations between pain and psychological burden. Spearman's bivariate correlations showed an association between pain severity and every psychological factor $(P \mathrm{~s}$ $\leq 0.01)$. Psychological factors were also highly intercorrelated $(P$ s $\leq 0.01)$. There was no association with years of MFS diagnosis and pain severity $\left(\mathrm{r}_{\mathrm{s}}=-0.10, P=0.18\right)$ or painrelated disability $\left(\mathrm{r}_{\mathrm{s}}=-0.03, P=0.72\right)$.

\section{Multivariate Correlates of Pain and Pain-Related Disability}

Pain severity.-Older individuals had increased pain severity when controlling for physical health and other demographic variables (see Table VI). Physical health independently accounted for $26 \%$ of the variance in pain severity. After controlling for demographic and physical health-related factors, pain-related disability, and pain catastrophizing were the only significant independent psychological variables associated with pain severity.

Pain-related disability.-Females, older individuals, and the unemployed had increased pain-related disability (see Table VII; $P$ s $\leq 0.01$ ). Physical health functioning was most strongly associated with pain-related disability, accounting for $39 \%$ of the variance when controlling for demographic characteristics. Even after controlling for demographic and 
physical health-related variables, mental health functioning remained significantly associated with pain-related disability.

\section{DISCUSSION}

This study sought to determine the clinical and psychological factors associated with pain and pain-related disability in MFS. Our results suggest that pain is a significant problem for these individuals, with pain averaging in the moderate range, and the majority report unremitting pain (70.3\%). Similar to previous findings, the most common site for pain was in the lower back, [Nelson et al., 2015; Rao et al., 2016] yet pain frequently spreads to numerous locations and becomes widespread for a subset of individuals. While physical health is the largest predictor of pain severity and pain-related disability, we find that pain catastrophizing and worse mental health functioning are significant correlates of either pain or pain-related disability, as has been demonstrated in numerous other painful conditions.

A unique contribution our findings make to the existing literature is the observation that individuals whose pain began early in the course of MFS have greater burden. This burden includes greater pain-related disability, pain catastrophizing, and depressive symptoms. Given the burden of pain to MFS patients [Rao et al., 2016], the impact throughout a patient's lifespan is substantial and affects emotional functioning, including fatigue [Bathen et al., 2014], sleep quality [Verbraecken et al., 2002], and social functioning [Peters et al., 2002]. We find that the participants who reported pain as a presenting symptom, as well as those who reported progression of pain to new locations, had profoundly worse psychological burden. As with other pain conditions, numerous studies have demonstrated that individuals with MFS report poor sleep, depressive symptoms, and worse mental health related factors [Peters et al., 2001b; Verbraecken et al., 2002; Rand-Hendriksen et al., 2010; Velvin et al., 2015a; Rao et al., 2016]. These symptoms are in part due to pain in MFS and consistent with the widely recognized overlap between pain and psychological burden in chronic musculoskeletal conditions. Given the stark differences in psychological burden for MFS individuals who had pain at syndrome onset and for individuals whose pain has spread since onset, additional investigation is needed to better understand the experience of pain in MFS, and to identify factors that predict the spread of pain and whether early intervention can mitigate long-term burden.

Many people who experience chronic pain also report disability in daily life related to their pain, including changes in activities of daily living, difficulties with mobility and ambulation, and changes in sleep and sexual activity. Our sample of individuals with MFS showed the expected relationship between greater pain-related disability and both worse mental health functioning and greater depressive symptom severity. Only worse mental health functioning (SF-12) predicted pain-related disability in multivariate analyses, independent of unemployment, physical health, and pain severity. The high correlation between depressive symptoms and mental health score $\left(r_{\mathrm{s}}=-0.73\right)$ and lack of independent effect of depressive symptoms in the final multivariate model suggests that mental health factors other than depressive symptoms (e.g., anxiety) may be contributing to pain-related disability. Mesfin et al. [2013] previously reported that the presence of dural ectasia in MFS was not associated with a significant increase in pain-related disability (ODI) scores over a 
10-year period. However, ours is the first study to report an association with pain-related disability using ODI and mental health in the MFS population.

Ratings of the severity of pain in MFS correlated with pain catastrophizing, an exaggerated negative cognitive-affective response to anticipated or actual pain that is associated with worse pain-related outcomes [Sullivan et al., 2001; Edwards et al., 2006; Quartana et al., 2009]. These scores were higher in various subgroups, including those reporting pain, those reporting pain at the time symptom onset, and those who had pain that spread from the initial site of pain. As is typically seen in other groups with chronically painful conditions, scores on pain catastrophizing were highly correlated with scores on the depressive symptom checklist $\left(\mathrm{r}_{\mathrm{s}}=0.67\right)$. The multivariate analyses confirmed the association with pain severity, even when unemployment, pain-related disability, depressive symptoms, and other potential covariates were controlled. While these are the first data in the literature on painrelated catastrophizing in MFS, previous reports indicate convergent effects. Peters et al. [2001b] demonstrated that chronically sore joints are associated with negative views of MFS and De Bie et al. [2004] showed pain severity is associated with greater psychological burden. Overwhelming evidence identifies catastrophizing and depressive symptoms as key risk factors for poor prognosis in patients with musculoskeletal pain conditions [Burns et al., 2003a,b; Edwards et al., 2011; Linton et al., 2011]. Depression and catastrophizing have been associated with greater pain severity among individuals with fibromyalgia, rheumatoid arthritis, osteoarthritis, and spinal pain. [Bousema et al., 2007; Rosemann et al., 2008; Wolff et al., 2008; Sullivan et al., 2009].

Our results indicate that pain severity, quality of pain, and associated symptoms of pain are retrospectively described as constant over the course of MFS. Significant improvements in life expectancy [Silverman et al., 1995; Willis et al., 2009] have transitioned focus on understanding pain and other symptoms in order to optimize quality of life [Velvin et al., 2016]. Similar to previous reports, pain quality is most frequently described as aching, sharp, stiff, dull, or throbbing [Nelson et al., 2015]. The most common symptoms associated with pain include stiffness, difficulty walking, muscle spasms, weakness, and increased sensitivity; thus the number of pain sites increases over the course of MFS, but pain quality does not change. The high prevalence of back pain in MFS has been associated with symptoms including scoliosis, dural ectasia, and vertebral body erosions [Ahn et al., 2001; Foran et al., 2005]. In our sample, participants with symptoms including dural ectasia, degenerative disc disease, kyphosis, and early osteoarthritis reported greater pain severity compared to participants without these symptoms.

A strength of our study was the moderate sample size and use of validated tools as outcome measures. Our overall incidence of symptoms of MFS, and reports of pain were consistent with previous reports supporting the generalizability of our study to individuals with MFS. One limitation of our methodology is the use of an anonymous online survey. We did not confirm diagnoses with a physician or medical records, but we did exclude in analyzes those who did not report a family history of MFS and did not report diagnoses by physical exam, cardiac or ophthalmologic exam. Use of an online survey may increase selection, classification, and respondent biases. In particular, our participants likely do not represent the worldwide MFS population, as our sample required internet access and is predominantly 
North American (90\%) and Non-Hispanic white. Studies indicate that ethnicity and sociocultural factors influence the experience of pain leading to differences in pain perception, self-care behaviors, and preferences for pain management [Merry et al., 2011; Campbell and Edwards, 2012]. As MFS affects all ethnic groups and both sexes, crosscultural differences on the impact of pain in MFS are not adequately examined based on our survey participants. Additionally, individuals with low literacy or psychiatric diagnoses, such as depression, may have less ability, motivation, or interest in taking an online survey and may therefore be under-represented. Finally, as this is a cross-sectional analysis we were unable to determine the temporal relationship or directionality of pain and psychological factors. Longitudinal studies are needed to evaluate the progression and natural history of pain manifestations in MFS and further studies are indicated to determine reproducibility of data using data from medical records.

\section{CONCLUSION}

Pain is a significant problem for individuals with MFS and is associated with profound disability and psychological burden. Pain may occur from the onset of MFS and frequently progresses throughout the body. Almost half of participants indicated that they had not had a pain diagnosis. This implies that a significant number of MFS patients may experience chronic pain but have not been evaluated and as a result not been afforded effective treatment. A possible reason for this is the fact that the musculoskeletal manifestations of MFS are less life threatening and as such receive less attention. Given the numerous and persistent pain experiences in this syndrome, MFS should be considered a chronic pain condition. With the growing population of older MFS patients the burden of chronic pain will become important and require treatment.

Longitudinal prospective studies are needed to better characterize the directionality of pain and psychological factors in individuals with MFS. Overwhelming evidence suggests that psychological factors can serve as prognostic indicators of enhanced disease progression, worsening physical disability, and reduced quality of life [Bousema et al., 2007; Edwards et al., 2011], while the perception of pain also promotes decrements in psychological functioning [Rosemann et al., 2008]. Given the substantial evidence that pain contributes to reduced physical and mental health in MFS, pain should be assessed at diagnosis and continuously monitored throughout the course of MFS. Pain management and psychological interventions are important considerations in the care of patients with MFS and should be included in a multidisciplinary approach in this patient population.

\section{ACKNOWLEDGMENTS} Work described in this manuscript was funded by grant NIH K23 NS070933 (CMC), grant NIH T32 NS070201
(VAM), and grant NIH T32 NS070201-12 (TJS).

Grant sponsor: NIH; Grant number: K23 NS070933 T32 NS070201 T32 NS070201-12. 


\section{REFERENCES}

Ahn NU, Nallamshetty L, Ahn UM, Buchowski JM, Rose PS, Garrett ES, Kebaish KM, Sponseller PD. 2001 Dural ectasia and conventional radiography in the Marfan lumbosacral spine. Skeletal Radiol 30:338-345.11465775

Bathen T, Velvin G, Rand-Hendriksen S, Robinson HS. 2014 Fatigue in adults with Marfan syndrome, occurrence and associations to pain and other factors. Am J Med Genet Part A 164A:19311939.24719044

Beck AT, Steer RA, Carbin MG. 1988 Psychometric properties of the Beck Depression Inventory: Twenty-five years of evaluation. Clin Psychol Rev 8:77-100.

Bousema EJ, Verbunt JA, Seelen HA, Vlaeyen JW, Knottnerus JA. 2007 Disuse and physical deconditioning in the first year after the onset of back pain. Pain 130:279-286.17467902

Burns JW, Glenn B, Bruehl S, Harden RN, Lofland K. 2003a Cognitive factors influence outcome following multidisciplinary chronic pain treatment: A replication and extension of a cross-lagged panel analysis. Behav Res Ther 41:1163-1182.12971938

Burns JW, Kubilus A, Bruehl S, Harden RN, Lofland K. 2003b Do changes in cognitive factors influence outcome following multidisciplinary treatment for chronic pain? A cross-lagged panel analysis. J Consult Clin Psychol 71:81-91.12602428

Campbell CM, Edwards RR. 2012 Ethnic differences in pain and pain management. Pain Manag 2:219-230.23687518

DasGupta A, Cai TT, Brown LD. 2001 Interval estimation for a binomial proportion. Statistical Science 16:101-133.

De Bie S, De Paepe A, Delvaux I, Davies S, Hennekam RC. 2004 Marfan syndrome in europe. Community Genet 7:216-225.15692197

Dean JC. 2007 Marfan syndrome: Clinical diagnosis and management. Eur J Hum Genet 15:724733.17487218

Dietz HC, Pyeritz RE. 1995 Mutations in the human gene for fibrillin-1 (FBN1) in the Marfan syndrome and related disorders. Hum Mol Genet 4:1799-1809.8541880

Dijkers M 2010 Comparing quantification of pain severity by verbal rating and numeric rating scales. J Spinal Cord Med 33:232-242.20737796

Edwards RR, Bingham CO, Bathon J, Haythornthwaite JA. 2006 Catastrophizing and pain in arthritis, fibromyalgia, and other rheumatic diseases. Arthritis Rheum 55:325-332.16583384

Edwards RR, Cahalan C, Calahan C, Mensing G, Smith M, Haythornthwaite JA. 2011 Pain, catastrophizing, and depression in the rheumatic diseases. Nat Rev Rheumatol 7:216224.21283147

Fairbank JC. 2000 The use of revised oswestry disability questionnaire. Spine (PhilaPa 1976) 25:2846-2847.

Farrar JT, Young JP, LaMoreaux L, Werth JL, Poole RM. 2001 Clinical importance of changes in chronic pain intensity measured on an 11-point numerical pain rating scale. Pain 94:149158.11690728

Foran JR, Pyeritz RE, Dietz HC, Sponseller PD. 2005 Characterization of the symptoms associated with dural ectasia in the Marfan patient. Am J Med Genet Part A 134A:58-65.15690402

Gandek B, Ware JE, Aaronson NK, Apolone G, Bjorner JB, Brazier JE, Bullinger M, Kaasa S, Leplege A, Prieto L, Sullivan M. 1998 Cross-validation of item selection and scoring for the SF-12 Health Survey in nine countries: Results from the IQOLA Project. International Quality of Life Assessment. J Clin Epidemiol 51:1171-1178.9817135

Grahame R, Pyeritz RE. 1995 The Marfan syndrome: Joint and skin manifestations are prevalent and correlated. Br J Rheumatol 34:126-131.7704457

Hasan A, Poloniecki J, Child A. 2007 Ageing in marfan syndrome. Int J Clin Pract 61:13081320.17627709

Hjermstad MJ, Fayers PM, Haugen DF, Caraceni A, Hanks GW, Loge JH, Fainsinger R, Aass N, Kaasa S, European Palliative Care Research C. 2011 Studies comparing numerical rating scales, 
verbal rating scales, and visual analogue scales for assessment of pain intensity in adults: A systematic literature review. J Pain Symptom Manage 41:1073-1093.21621130

Iams HD. 2010 Diagnosis and management of Marfan syndrome. Curr Sports Med Rep 9:9398.20220350

Jenkinson C, Layte R, Jenkinson D, Lawrence K, Petersen S, Paice C, Stradling J. 1997 A shorter form health survey: Can the SF-12 replicate results from the SF-36 in longitudinal studies? J Public Health Med 19:179-186.9243433

Jensen MP, McFarland CA. 1993 Increasing the reliability and validity of pain intensity measurement in chronic pain patients. Pain 55:195-203.8309709

Linton SJ, Nicholas MK, MacDonald S, Boersma K, Bergbom S, Maher C, Refshauge K. 2011 The role of depression and catastrophizing in musculoskeletal pain. Eur J Pain 15:416-422.20884261

Loeys BL, Dietz HC, Braverman AC, Callewaert BL, De Backer J, Devereux RB, Hilhorst-Hofstee Y, Jondeau G, Faivre L, Milewicz DM, Pyeritz RE, Sponseller PD, Wordsworth P, De Paepe AM. 2010 The revised Ghent nosology for the Marfan syndrome. J Med Genet 47:476-485.20591885

Merry B, Campbell CM, Buenaver LF, McGuire L, Haythornthwaite JA, Doleys DM, Edwards RR. 2011 Ethnic group differences in the outcomes of multidisciplinary pain treatment. J Musculoskelet Pain 19:24-30.21731407

Mesfin A, Ahn NU, Carrino JA, Sponseller PD. 2013 Ten-year clinical and imaging follow-up of dural ectasia in adults with Marfan syndrome. Spine J 13:62-67.23218825

Nelson AM, Walega DR, McCarthy RJ. 2015 The incidence and severity of physical pain symptoms in marfan syndrome: A survey of 993 patients. Clin J Pain 31:1080-1086.25565586

Pearson GD, Devereux R, Loeys B, Maslen C, Milewicz D, Pyeritz R, Ramirez F, Rifkin D, Sakai L, Svensson L, Wessels A, Van Eyk J, Dietz HC, National Heart L, Blood I, National Marfan Foundation Working G, 2008 Report of the national heart, lung, and blood institute and national marfan foundation working group on research in marfan syndrome and related disorders. Circulation 118:785-791.18695204

Peters KF, Horne R, Kong F, Francomano CA, Biesecker BB. 2001a Living with Marfan syndrome II. Medication adherence and physical activity modification. Clin Genet 60:283-292.11683774

Peters KF, Kong F, Hanslo M, Biesecker BB. 2002 Living with Marfan syndrome III. Quality of life and reproductive planning. Clin Genet 62:110-120.12220448

Peters KF, Kong F, Horne R, Francomano CA, Biesecker BB. 2001b Living with Marfan syndrome I. Perceptions of the condition. Clin Genet 60:273-282.11683773

Quartana PJ, Campbell CM, Edwards RR. 2009 Pain catastrophizing: A critical review. Expert Rev Neurother 9:745-758.19402782

Ramirez F, Dietz HC. 2007 Marfan syndrome: From molecular pathogenesis to clinical treatment. Curr Opin Genet Dev 17:252-258.17467262

Rand-Hendriksen S, Johansen H, Semb SO, Geiran O, Stanghelle JK, Finset A. 2010 Health-related quality of life in Marfan syndrome: A cross-sectional study of Short Form 36 in 84 adults with a verified diagnosis. Genet Med 12:517-524.20613543

Rao SS, Venuti KD, Dietz HC, Sponseller PD. 2016 Quantifying health status and function in Marfan syndrome. J Surg Orthop Adv 25:34-40.27082886

Rosemann T, Laux G, Szecsenyi J, Wensing M, Grol R. 2008 Pain and osteoarthritis in primary care: Factors associated with pain perception in a sample of 1,021 patients. Pain Med 9:903910.18702636

Silverman DI, Burton KJ, Gray J, Bosner MS, Kouchoukos NT, Roman MJ, Boxer M, Devereux RB, Tsipouras P. 1995 Life expectancy in the Marfan syndrome. Am J Cardiol 75:157-160.7810492

Sullivan M, Tanzer M, Stanish W, Fallaha M, Keefe FJ, Simmonds M, Dunbar M. 2009 Psychological determinants of problematic outcomes following Total Knee Arthroplasty. Pain 143:123129.19304392

Sullivan MJL, Bishop SR, Pivik J. 1995 The pain catastrophizing scale: Development and validation. Psychol Assess 7:524-532.

Sullivan MJL, Thorn B, Haythornthwaite JA, Keefe F, Martin M, Bradley LA, Lefebvre JC. 2001 Theoretical perspectives on the relation between catastrophizing and pain. Clin J Pain 17:5264.11289089 
Van Tongerloo A, De Paepe A. 1998 Psychosocial adaptation in adolescents and young adults with Marfan syndrome: An exploratory study. J Med Genet 35:405-409.9610804

Velvin G, Bathen T, Rand-Hendriksen S, Geirdal AO. 2015a Systematic review of the psychosocial aspects of living with Marfan syndrome. Clin Genet 87:109-116.24813698

Velvin G, Bathen T, Rand-Hendriksen S, Geirdal AO. 2015b Work participation in adults with Marfan syndrome: Demographic characteristics, MFS related health symptoms, chronic pain, and fatigue. Am J Med Genet Part A 167A:3082-3090.26420568

Velvin G, Bathen T, Rand-Hendriksen S, Geirdal AO. 2016 Systematic review of chronic pain in persons with Marfan syndrome. Clin Genet 89:647-658.26607862

Verbraecken J, Declerck A, Van de Heyning P, De Backer W, Wouters EFM. 2002 Evaluation for sleep apnea in patients with Ehlers-Danlos syndrome and Marfan: A questionnaire study. Clin Genet 60:360-365.

von Kodolitsch Y, De Backer J, Schuler H, Bannas P, Behzadi C, Bernhardt AM, Hillebrand M, Fuisting B, Sheikhzadeh S, Rybczynski M, Kolbel T, Puschel K, Blankenberg S, Robinson PN. 2015 Perspectives on the revised Ghent criteria for the diagnosis of Marfan syndrome. Appl Clin Genet 8:137-155.26124674

Ware J, Kosinski M, Keller SD. 1996 A 12-Item Short-Form Health Survey: Construction of scales and preliminary tests of reliability and validity. Med Care 34:220-233.8628042

Willis L, Roosevelt GE, Yetman AT. 2009 Comparison of clinical characteristics and frequency of adverse outcomes in patients with Marfan syndrome diagnosed in adulthood versus childhood. Pediatr Cardiol 30:289-292.19184183

Wolff B, Burns JW, Quartana PJ, Lofland K, Bruehl S, Chung OY. 2008 Pain catastrophizing, physiological indexes, and chronic pain severity: Tests of mediation and moderation models. $\mathrm{J}$ Behav Med 31:105-114.18158618

Yu DS. 2010 Insomnia severity index: Psychometric properties with chinese community-dwelling older people. J Adv Nurs 66:2350-2359.20722803 


\section{TABLE I.}

\section{Demographic Information}

\begin{tabular}{lc}
\hline Group & MFS (N= 245) \\
Sex ( $\%$ female) & $73.3 \%$ \\
$\begin{array}{l}\text { Age in years, mean } \\
\text { (range) }\end{array}$ & $35-44(18-75)$ \\
Ethnicity & \\
$\quad$ Non-Hispanic white & $89.3 \%$ \\
$\quad$ Asian American & $3.3 \%$ \\
$\quad$ African American & $4.1 \%$ \\
$\quad$ Other & $3.3 \%$ \\
North American resident & $90.2 \%$ \\
Employment status & $43.4 \%$ \\
$\quad$ unemployed) & \\
Average years since & $19.9(15.4)$ \\
diagnosis (SD) & \\
Average number of MFS & $11.7(5.5)$ \\
symptoms ${ }^{\text {(SD) }}$ & \\
\hline
\end{tabular}

$\mathrm{SD}=$ standard deviation;

${ }^{a}$ MFS symptoms included in survey: aortic dilatation, arachnodactyly, gastroesophageal reflux, bicuspid aortic valve, cysts, cystic medial necrosis, degenerative disc disease, deviated septum, dural ectasia, early cataracts, early glaucoma, early osteoarthritis, ectopic lentis, emphysema, iris coloboma, above average height, heart palpitations, hernias, high palate, joint hypermobility, kyphosis, heart murmur, malocclusion, micrognathia, mitral valve prolapse, myopia, obstructive lung disease, osteopenia, osteoporosis, pectus carinatum/pectus excavatum, pes planus, pneumothorax, retinal detachment, scoliosis, sleep apnea, stretch marks, temporomandibular joint disorder. 


\section{TABLE II.}

Frequency of Self-Reported Physical Features of MFS

\begin{tabular}{|c|c|c|c|c|}
\hline \multirow{2}{*}{$\begin{array}{l}\text { I. Physical Features } \\
\text { MFS }(\mathrm{N}=\mathbf{2 4 5})\end{array}$} & \multicolumn{2}{|c|}{$\begin{array}{l}\text { Presenting } \\
\text { symptoms }\end{array}$} & \multicolumn{2}{|c|}{ Current symptoms } \\
\hline & $(\%)$ & {$[\mathrm{CI}]$} & $(\%)$ & {$[\mathrm{CI}]$} \\
\hline $\begin{array}{c}\text { Cardiovascular } \\
\text { symptoms }^{a}\end{array}$ & 29.0 & $23.6-34.9$ & 93.1 & $89.4-95.7$ \\
\hline Aortic dilatation $^{e}$ & 11.4 & $7.9-15.9$ & 62.4 & $56.3-68.3$ \\
\hline Ocular symptoms $b$ & 19.6 & $15.0-24.9$ & 72.2 & $66.4-77.6$ \\
\hline Ectopic lentis ${ }^{e}$ & 7.3 & $4.6-11.1$ & 12.7 & $8.9-17.2$ \\
\hline $\begin{array}{l}\text { Musculoskeletal } \\
\text { symptoms }\end{array}$ & 36.3 & $30.5-42.5$ & 95.5 & $92.4-97.6$ \\
\hline Pain $^{d}$ & 28.2 & $22.8-34.0$ & 89.0 & $84.6-92.4$ \\
\hline
\end{tabular}

[CI], 95\% confidence interval;

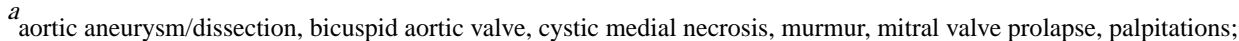

$b$

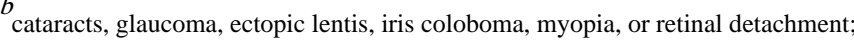

${ }^{c}$ above average height, arachnodactylyl, joint hypermobility, kyphosis, scoliosis, degenerative disk disease, early-onset osteoarthritis, osteopenia, osteoporosis, pectus carinatum/excavatum, or pes planus;

$d_{\text {pain, ache, hurt, or discomfort; }}$

$e_{\text {cardinal feature of MFS. }}$ 


\section{TABLE III.}

Current Numeric Pain Rating (NRS) by MFS Symptom

\begin{tabular}{lccccccccc}
\hline & \multicolumn{4}{c}{ No symptom } & \multicolumn{7}{c}{ Presence of symptom } \\
& $\mathbf{N}$ & $\mathbf{M} \pm \mathbf{S D}$ & $\mathbf{M D N}(\mathbf{I Q R})$ & $\mathbf{N}$ & $\mathbf{M} \pm \mathbf{S D}$ & $\mathbf{M D N}(\mathbf{I Q R})$ & \multicolumn{1}{c}{$\Delta \mathbf{M D N}[\mathbf{C I}]$} & $\mathbf{z}$ \\
Kyphosis & 148 & $4.0 \pm 2.6$ & $4(2-6)$ & 38 & $5.9 \pm 2.3$ & $6(4.75-8)$ & $-2(-3$ to -1$)$ & $-4.3^{* *}$ \\
Degenerative disc disease & 129 & $3.9 \pm 2.6$ & $4(2-6)$ & 57 & $5.5 \pm 2.4$ & $6(4-7)$ & $-2(-3$ to -1$)$ & $-4.0^{* *}$ \\
Early osteoarthritis & 134 & $3.9 \pm 2.7$ & $4(2-6)$ & 52 & $5.4 \pm 2.2$ & $6(4-7)$ & $-2(-3$ to -1$)$ & $-3.6^{* *}$ \\
Dural ectasia & 127 & $4.1 \pm 2.8$ & $4(2-6)$ & 59 & $5.0 \pm 2.3$ & $6(3-7)$ & $-1(-2$ to 0$)$ & $-2.5^{*}$ \\
Scoliosis & 89 & $4.1 \pm 2.7$ & $4(2-6)$ & 97 & $4.6 \pm 2.6$ & $5(3-7)$ & $-1(-1$ to 0$)$ & -1.3 \\
Joint hypermobility & 58 & $4.0 \pm 2.8$ & $4(2-6)$ & 128 & $4.5 \pm 2.6$ & $5(3.25-6)$ & $-1(-1$ to 0$)$ & -1.2 \\
\hline
\end{tabular}

$\mathrm{N}$, number of respondents; $\mathrm{M} \pm \mathrm{SD}$, mean and standard deviation; $\mathrm{MDN}(\mathrm{IQR})$, median (interquartile range); $\triangle \mathrm{MDN}$, difference in median between groups; $[\mathrm{CI}], 95 \%$ confidence interval of difference between means; z, Mann-Whitney test.

${ }^{*} \leq 0.01$,

*** $P<0.001$ 


\section{TABLE IV.}

Pain and Psychological Burden in MFS Groups

\begin{tabular}{|c|c|c|c|c|c|c|}
\hline & $\operatorname{MFS}(N=147)$ & $\begin{array}{l}\text { Has pain } \\
(N=142)\end{array}$ & $\begin{array}{l}\text { Pain not a } \\
\text { presenting sx } \\
(N=94)\end{array}$ & $\begin{array}{c}\text { Pain as a } \\
\text { presenting sx } \\
\quad(N=53)\end{array}$ & $\begin{array}{l}\text { Pain has not spread } \\
\text { since onset } \\
(\mathbf{N}=\mathbf{5 8})\end{array}$ & $\begin{array}{l}\text { Pain has spread } \\
\text { since onset } \\
(N=89)\end{array}$ \\
\hline Pain severity & $2.5(2.0-3.3)$ & $2.8(2.3-3.5)$ & $2.5(2.0-3.3)$ & $3.0(2.3-3.5)$ & $2.3(1.8-3.0)$ & $3.0^{* *}(2.3-3.5)$ \\
\hline $\begin{array}{c}\text { Pain-related } \\
\text { disability }\end{array}$ & $36.0(20.0-51.1)$ & $37.9(24.0-52.0)$ & $34.0(20.0-49.2)$ & $37.9^{*}(25.3-61.0)$ & $24.0(16.0-36.0)$ & $46.0^{* * *}(35.0-60.0)$ \\
\hline Catastrophizing & $13.0(7.0-25.0)$ & $16(8.0-28.5)$ & $14.0(8.0-23.3)$ & $23.0 *(9.0-32.0)$ & $11.5(7.0-17.8)$ & $21.0^{* *}(10.0-32.0)$ \\
\hline Physical health & $30.2(24.9-38.3)$ & $29.8(24.7-36.7)$ & $31.3(25.1-39.6)$ & $29.3(23.3-35.5)$ & $36.1(31.1-43.5)$ & $26.7^{* *}(23.0-33.4)$ \\
\hline Mental health & $40.5(31.5-47.8)$ & $40.1(30.5-47.3)$ & $42.1(32.8-48.0)$ & $36.8(28.5-45.8)$ & $42.2(35.1-48.7)$ & $39.3(29.4-47.3)$ \\
\hline $\begin{array}{l}\text { Insomnia } \\
\quad \text { symptoms }\end{array}$ & $11.0(7.0-15.0)$ & $12.0(8.0-16.5)$ & $11.6(8.0-15.3)$ & $12.0(8.6-17.0)$ & $10.5(7.0-13.0)$ & $13.0^{* *}(9.0-17.8)$ \\
\hline $\begin{array}{l}\text { Depressive } \\
\text { symptoms }\end{array}$ & $14.0(7.5-20.5)$ & $16.0(10.0-23.0)$ & $15.0(9.0-23.0)$ & $19.0 *(11.0-25.5)$ & $13.0(9.0-20.8)$ & $17.0^{*}(11.0-24.0)$ \\
\hline
\end{tabular}

Data are presented as median and interquartile range (IQR).

${ }^{a}$ Significance between pain as presenting symptom or not;

$b_{\text {significance between pain spread or not. }}$

${ }^{*} P \unlhd 01, * * * *$

** $P<0.001$ 
TABLE V.

Spearman Correlation Matrix Between Pain, Physical Health, and Psychological Measures for MFS Individuals $^{a}$

\begin{tabular}{lcccccc}
\hline & $\mathbf{1}$ & $\mathbf{2}$ & $\mathbf{3}$ & $\mathbf{4}$ & $\mathbf{5}$ & $\mathbf{6}$ \\
1. Pain severity $(\mathrm{N}=177)$ & 1.0 & & & & & \\
2. Pain-related disability $(\mathrm{N}=171)$ & $0.65^{* *}$ & 1.0 & & & & \\
3. Catastrophizing $(\mathrm{N}=147)$ & $0.45^{* * *}$ & $0.46^{* *}$ & 1.0 & & & \\
4. Physical health $(\mathrm{N}=198)$ & $-0.56^{* *}$ & $-0.77^{* *}$ & $-0.24^{*}$ & 1.0 & & \\
5. Mental health $(\mathrm{N}=147)$ & $-0.24^{*}$ & $-0.31^{* *}$ & $-0.43^{* *}$ & -0.06 & 1.0 & \\
6. Insomnia symptoms $(\mathrm{N}=176)$ & $0.40^{* * *}$ & $0.48^{* *}$ & $0.51^{* *}$ & $-0.36^{* *}$ & $-0.37^{* *}$ & 1.0 \\
7. Depressive symptoms $(\mathrm{N}=176)$ & $0.35^{* * *}$ & $0.44^{* *}$ & $0.67^{* *}$ & -0.19 & $-0.73^{* *}$ & $0.58^{* *}$ \\
\hline
\end{tabular}

Includes only the subgroup who reported having pain.

* $P \unlhd 0.01$,

** $P<0.001$ 
TABLE VI.

Hierarchical Linear Analysis of Pain Severity for MFS Individuals Reporting Pain

\begin{tabular}{|c|c|c|c|c|c|c|}
\hline Step Variable & $\beta$ & SE & Beta & $\mathbf{t}$ & $\mathbf{R}^{2}$ & $\Delta \mathbf{R}^{2}$ \\
\hline 1. Sex & -0.22 & 0.17 & -0.11 & -1.30 & & \\
\hline Age & 0.00 & 0.05 & 0.01 & 0.06 & & \\
\hline Ethnicity & -0.08 & 0.14 & -0.05 & -0.60 & & \\
\hline Employment & 0.28 & 0.14 & 0.17 & 1.95 & 0.04 & 0.04 \\
\hline 2. Sex & -0.07 & 0.15 & -0.04 & -0.45 & & \\
\hline Age & -0.13 & 0.05 & -0.25 & $-2.97^{*}$ & & \\
\hline Ethnicity & -0.03 & 0.12 & -0.02 & -0.28 & & \\
\hline Employment & 0.07 & 0.13 & 0.04 & 0.56 & & \\
\hline Physical health & -0.05 & 0.01 & -0.60 & $-7.03^{* *}$ & 0.30 & 0.26 \\
\hline 3. Sex & 0.02 & 0.13 & 0.01 & 0.16 & & \\
\hline Age & -0.05 & 0.05 & -0.08 & -1.02 & & \\
\hline Ethnicity & -0.14 & 0.11 & -0.09 & -1.32 & & \\
\hline Employment & -0.11 & 0.12 & -0.07 & -0.94 & & \\
\hline Physical health & -0.01 & 0.01 & -0.13 & -1.07 & & \\
\hline Pain-related disability & 0.02 & 0.01 & 0.49 & $4.11^{* *}$ & & \\
\hline Catastrophizing & 0.02 & 0.01 & 0.28 & $3.25^{*}$ & & \\
\hline Mental health & 0.00 & 0.01 & -0.02 & -0.19 & & \\
\hline Insomnia symptoms & 0.01 & 0.01 & 0.06 & 0.67 & & \\
\hline Depressive symptoms & -0.01 & 0.01 & -0.13 & -1.25 & 0.48 & 0.19 \\
\hline
\end{tabular}


TABLE VII.

Hierarchical Linear Analysis of Pain Severity for MFS Individuals Reporting Pain

\begin{tabular}{|c|c|c|c|c|c|c|}
\hline Step Variable & $\beta$ & SE & Beta & $\mathbf{t}$ & $\mathbf{R}^{2}$ & $\Delta \mathbf{R}^{2}$ \\
\hline 1. Sex & -9.96 & 3.62 & -0.21 & $-2.75^{*}$ & 0.21 & 0.21 \\
\hline Age & 2.64 & 1.01 & 0.21 & $2.60^{*}$ & & \\
\hline Ethnicity & 1.42 & 2.89 & 0.04 & 0.49 & & \\
\hline Employment & 12.82 & 3.04 & 0.34 & $4.22^{* *}$ & & \\
\hline 2. Sex & -5.47 & 2.61 & -0.12 & -2.10 & & \\
\hline Age & -1.32 & 0.80 & -0.10 & -1.65 & & \\
\hline Ethnicity & 2.84 & 2.06 & 0.08 & 1.38 & & \\
\hline Employment & 6.84 & 2.23 & 0.18 & $3.07^{*}$ & & \\
\hline Physical health & -1.43 & 0.13 & -0.73 & $-11.47^{* *}$ & 0.60 & 0.39 \\
\hline 3. Sex & -4.67 & 2.13 & -0.10 & -2.19 & & \\
\hline Age & 0.75 & 0.72 & 0.06 & 1.05 & & \\
\hline Ethnicity & 1.22 & 1.71 & 0.03 & 0.72 & & \\
\hline Employment & 2.43 & 1.89 & 0.06 & 1.29 & & \\
\hline Physical health & -1.16 & 0.13 & -0.59 & $-8.94^{* *}$ & & \\
\hline Pain Severity & 5.51 & 1.34 & 0.24 & $4.11^{* *}$ & & \\
\hline Catastrophizing & 0.09 & 0.09 & 0.06 & 1.02 & & \\
\hline Mental health & -0.54 & 0.12 & -0.31 & $-4.58^{* *}$ & & \\
\hline Insomnia symptoms & 0.02 & 0.21 & 0.00 & 0.07 & & \\
\hline Depressive symptoms & -0.07 & 0.14 & -0.04 & -0.53 & 0.76 & 0.15 \\
\hline
\end{tabular}

